\title{
Dynamic and Facilitated Binding of Topoisomerase Accelerates Topological Relaxation
}

\author{
D. Michieletto ${ }^{1,2, *}$ Y. A. G. Fosado, ${ }^{1, \dagger}$ E. Melas, ${ }^{1}$ M. Baiesi, ${ }^{3,4}$ L. Tubiana, ${ }^{5,6,7}$ and E. Orlandini ${ }^{3,4}$ \\ ${ }^{1}$ School of Physics and Astronomy, University of Edinburgh, \\ Peter Guthrie Tait Road, Edinburgh, EH9 3FD, UK \\ ${ }^{2} M R C$ Human Genetics Unit, Institute of Genetics and Cancer, \\ University of Edinburgh, Edinburgh EH4 $2 X U$, UK \\ ${ }^{3}$ Department of Physics and Astronomy, University of Padova, Via Marzolo 8, I-35131 Padova, Italy \\ ${ }^{4}$ INFN, Sezione di Padova, Via Marzolo 8, I-35131 Padova, Italy \\ ${ }^{5}$ Physics Department, University of Trento, via Sommarive, 14 I-38123 Trento, Italy \\ ${ }^{6}$ INFN-TIFPA, Trento Institute for Fundamental Physics and Applications, I-38123 Trento, Italy \\ ${ }^{7}$ Faculty of Physics, University of Vienna, Boltzmanngasse 5, 1090 Vienna, Austria
}

How type 2 Topoisomerase (TopoII) proteins relax and simplify the topology of DNA molecules is one of the most intriguing open questions in biophysics. Most of the existing models neglect the dynamics of TopoII which is characteristics for proteins searching their targets via facilitated diffusion. Here, we show that dynamic binding of TopoII speeds up the topological relaxation of knotted substrates by enhancing the search of the knotted arc. Intriguingly, this in turn implies that the timescale of topological relaxation is virtually independent of the substrate length. We then discover that considering binding biases due to facilitated diffusion on looped substrates steers the sampling of the topological space closer to the boundaries between different topoisomers yielding an optimally fast topological relaxation. We discuss our findings in the context of topological simplification in vitro and in vivo.

The topological regulation of DNA in vivo is one of the most important biophysical processes and allow genomes to function and cells to multiply. The crucial role of TopoII to reduce the topological complexity of naked DNA in vitro is now well established [1]. At the same time, recent works strongly suggest that the genome is itself surprisingly topologically simple in vivo [2-4]. These fascinating findings have recently been partially rationalized in terms of a synergistic interaction of TopoII with Structural Maintenance of Chromosomes (SMC) proteins $[5,6]$. However an accurate explanation of TopoIIonly regulation is still lacking.

Most of the existing polymer models of DNA-TopoII interaction are in equilibrium and mimic TopoII-bound regions by allowing inter-segment crossing at the expense of a modest amount of energy (comparable with the thermal one); additionally, they do not either account for TopoII binding/unbinding kinetics or place TopoII at random (or even everywhere) along the DNA [7, 8]. A notable exception is the class of models accounting for hooked juxtapositions that violate detailed balance [912]. In spite of this, these models do not consider either the kinetics or any form of bias in the binding pattern of TopoII. Motivated by this, we specifically investigate the effect of dynamic binding of TopoII and its transitory biases towards regions of large local density due, for instance, to facilitated diffusion on locally looped substrates $[13,14]$.

The key discovery of this work is that both fast (un)binding kinetics and biased binding dramatically shorten the timescale over which knotted substrates are relaxed to their steady state. By using a simplified 2D random walk model on idealised knot spaces, we ar- gue that dynamic and biased binding enhance the sampling between knot types thereby speeding up the unknotting process. Importantly, thanks to the (sublinear) scaling behaviour of the knotted region with polymer length $[15,16]$, these binding mechanisms also naturally yield a rate of topological relaxation that is only weakly dependent on the substrate length.

Results. We model a torsionally relaxed (nicked) DNA plasmid $3.6 \mathrm{kbp}$-long as a bead-spring polymer made of 500 beads with size $\sigma=2.5 \mathrm{~nm}=7.3 \mathrm{bp}$ and connected by finitely extensible springs (see SI) and persistence length $l_{p}=50 \mathrm{~nm}$. Beads interact via a purely steric Lennard-Jones repulsion while the TopoII-bound segment is modelled as a 50 beads ( $L=360 \mathrm{bp}$ )-long segment (shorter segments yield qualitative similar results but display slower kinetics) displaying soft interactions with the other beads thereby allowing the TopoII-bound segment to undergo strand-crossing with a small energy penalty $\left(2 k_{B} \mathrm{~T}\right)$. We dynamically update the position of the TopoII segment following different rules: either randomly or with a tendency to move towards certain local geometric features (see below). Unless otherwise stated, we study the relaxation of the knotting probability to its steady state by starting from a thermally equilibrated polymer tied in a $5_{1}$ torus knot. The simulations are performed in LAMMPS [17] using implicit solvent and coupled to custom-made $\mathrm{C}++$ codes to perform the dynamic update of the TopoII region. The averages of this relaxation process are performed over at least 64 independent replicas where we follow the topology of the polymer by computing its Alexander determinant using the kymoknot software [18].

To model the (un)binding kinetics of TopoII (from) 


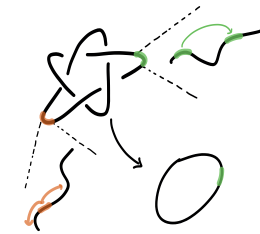

a
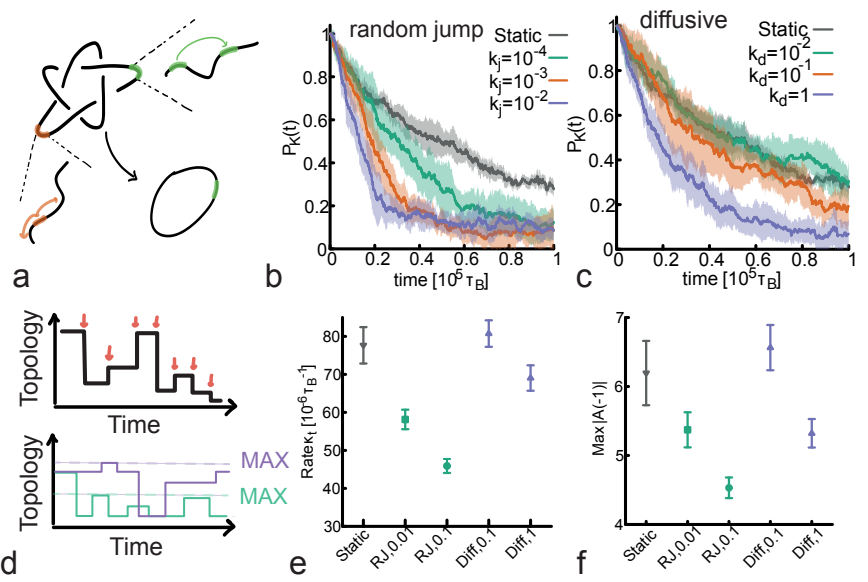

d

e

Figure 1. Unknotting is faster for dynamic TopoII. a Sketch of the system: one TopoII remains bound to the DNA and changes location by either random jumps (green) at rate $k_{j}$ (units of $\tau_{B}^{-1}$ ) or via diffusion (orange) at rate $k_{d}$ (units of $\left.\sigma^{2} / \tau_{B}\right)$. b The larger the jumping rate of TopoII, the faster the relaxation of $P_{K}(t)$. c The relaxation of $P_{K}(t)$ is also faster for more diffusive TopoII, albeit the effect is milder than in b. d Sketch of how we compute $\kappa_{t}$ and $\operatorname{Max}|A(-1)|$. e $\kappa_{t}$ is model and rate dependent and decreases for larger jumping/diffusion rates. $\mathbf{f} \operatorname{Max}|A(-1)|$ also decreases for more dynamic binding or faster diffusion. Smaller $\kappa_{t}$ and $\operatorname{Max}|A(-1)|$ indicate slower but more precise evolution of topology towards the steady state, overall rendering the simplification process more efficient.

to a DNA substrate, we consider a simplified scenario in which there is always one TopoII bound, and analyse the relaxation of the knotting probability for different rates at which TopoII is relocated. For a random (un)binding process, this is equivalent to the situation in which TopoII randomly "jumps" at rate $k_{j}$ (with $k_{j}=0$ being the static case) measured in (inverse) Brownian times $\tau_{B}=\sigma^{2} / D_{0}$, where $D_{0}$ is the diffusion coefficient of a bead. From our simulations, a rate as little as $k_{j}=10^{-4} \tau_{B}^{-1}$ already appears to give a significant deviation from the static case. Rates equal, or larger than, $k_{j}=10^{-2} \tau_{B}^{-1}$ have reached the maximum relaxation efficiency (see Fig. 1b). Since the longest relaxation time of the chain under consideration $(N=500$ beads and $l_{p}=20 \sigma$ in this figure) is about $10^{5} \tau_{B}$ (see SM), it means that about 10 "jumps" within one relaxation time are enough to significantly speed up the topological simplification with respect to a static TopoII.

Motivated by the notion of facilitated diffusion, namely a target search made by alternated rounds of 3D and 1D diffusion [14, 19-21], we also test whether and to which extent a purely curvilinear (1D) diffusion of TopoII impacts on the topological simplification rate. Similarly to the case of random jumps, we update the position of the only TopoII bound by sliding it randomly along the substrate every $k_{d}^{-1}$ time step, i.e. with a diffusion constant $D=0.5 k_{d} \sigma^{2}$. We find that larger diffusion rates $\left(k_{d} \geq \tau_{B}^{-1}\right)$ are needed to relax as efficiently as random jumps (Fig. 1c).

To understand this enhancement in topological simplification, we compute the rate $\kappa_{t}$ at which topologychanging strand-crossing operations occur, without discriminating between events that reduce or increase the knot complexity (this observable is schematically shown in Fig. 1d(top)). In Fig. 1e one can appreciate that both the diffusive and jump models yield a smaller $\kappa_{t}$ compared to the static one. Thus, interestingly, dynamic binding of TopoII gives rise to processes in which the knot type of the substrate changes less frequently than in the case of static TopoII.

To quantify how widely the substrates explore the attainable topological space we measure the ensemble average of the maximum value attained by the Alexander determinant evaluated at -1 (known as knot determinant). This is done for each replica of the system (denoted as $\operatorname{Max}|A(-1)|$ and excluding the initial $55_{1}$ topology, see Fig. 1d for a sketch). Note that $\operatorname{Max}|A(-1)|$ depends on the underlying binding process and the corresponding rates (Fig. 1F). In particular, it is smaller for fast diffusion and for moderately fast (compared with the Brownian time) random jumps.

In summary, the behavior of $\kappa_{t}$ and $\operatorname{Max}|A(-1)|$ suggest that dynamic binding of TopoII yields more precise relaxation pathways involving fewer topology-changing strand-crossings. This may be explained as follows: once TopoII lands on a segment embedded within a physically knotted region (or knotted arc) [15, 16], there is an entropic gain in relaxing the substrate to its topological equilibrium. On the other hand, if TopoII lands on a region that is not physically knotted and sufficiently swollen, then the chances of generating a topologychanging strand crossing in a short time (before TopoII moves somewhere else) is low. Furthermore, the limited residence time hinders repeated topology-changing strand-crossing events. This suggests that a key adimensional parameter of the topological simplification process is the ratio between the timescale of residency of TopoII, $\tau_{T}=1 / k_{j}$, and the relaxation (Rouse) time of the TopoII-bound polymer segment $\tau_{R}$; when $\tau_{T}<\tau_{R}$ we expect the dynamic binding to preferentially drive topological simplification.

Finally, it is worth mentioning that in static models of TopoII, the rate-limiting step for the topological evolution of the substrate is the diffusion dynamics of the knotted region (i.e. the knot has to find the TopoII-bound segment). In analogy with reptation polymer dynamics, this process scales as $\sim\left(N-l_{k}\right)^{2} / D_{k}$ with $l_{k}$ being the length of the knot and $D_{k} \simeq D_{0} / l_{k}$ its curvilinear diffusion $[22,23]$. On the other hand, for dynamic TopoII, it is the protein that "finds" the knot first, in a much smaller timescale $\lesssim N / k_{j}$.

Topological Relaxation with Biased TopoII. Motivated by the fact that TopoII has been proposed to co- 

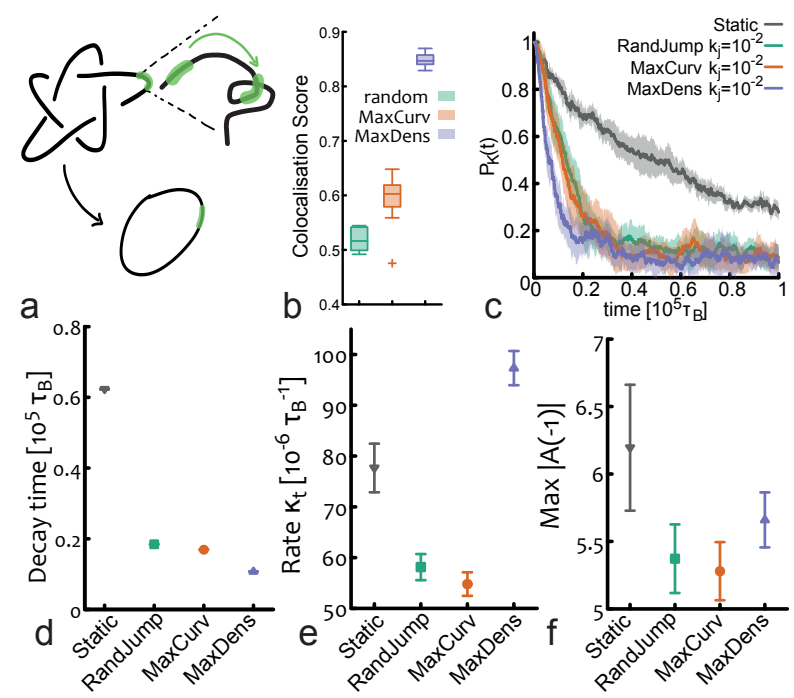

Figure 2. TopoII binding to Regions of Local Maximum Density is the Most Efficient Simplification Pathway. a Sketch of the model. b Boxplot showing that, on a $5_{1}$ knot spanning about half of the contour length of polymer, the bead with maximum local density is more than $80 \%$ of the times colocalised with the knotted arc, in agreement with the weakly localised nature of the knot. c Knotting probability for different models and same jumping rate. $\mathbf{d}$ Decay time $\tau$ obtained from an exponential fitting $e^{-t / \tau}$ of the knotting probability curves in $\mathbf{c}$ at early times. e Rate of topology-changing operations. f Average maximum value of Alexander determinant at -1 .

localise with DNA regions of large curvature [24], we explore a model in which the protein binds preferentially to regions with high bending. Additionally, we also investigate the case in which TopoII preferentially binds regions of large local density. These two binding modes are motivated by the fact that, in general, any protein that displays a weak non-specific binding affinity to DNA is expected to undergo facilitated diffusion [21] which can create transient binding biases to regions of crumpled or looped DNA [14] (i.e. with large density) or, as we argue here, knotted DNA due to its (weakly) localised [25], or even metastable tight [26], nature.

To couple the binding dynamic process with these geometric features (i.e. curvature and local density) we define the region of maximum local curvature as the portion of the polymer around the bead of index $i_{\text {maxcurv }}$ such that $i_{m c}=$ $\operatorname{argmax}_{i}\left[\sum_{j=-l_{T} / 2}^{l_{T} / 2} 1-\left(\boldsymbol{t}_{i+j} \boldsymbol{t}_{i+j+1}\right) /\left(\left|\boldsymbol{t}_{i+j}\right|\left|\boldsymbol{t}_{i+j+1}\right|\right)\right]$ where $l_{T}=50$ is the length of the TopoII region, $\boldsymbol{t}_{i} \equiv \boldsymbol{r}_{i+1}-\boldsymbol{r}_{i}$ is the tangent vector at bead $i$ (periodic conditions on the index $j$ are implicit). Similarly, the region of maximum local density is defined as $i_{m d}=\operatorname{argmax}_{i}\left\{\sum_{j=1}^{N} \Theta\left(R-\left|\boldsymbol{r}_{i}-\boldsymbol{r}_{j}\right|\right)\right\}$ where $\Theta(x)=1$ if $x>0$ and 0 otherwise and we take $R=50^{\nu}$ with $\nu=0.588$ as expected for a chain with excluded volume interaction [23]. Interestingly we find that for a $5_{1}$ topology spanning about $50 \%$ of the polymer
$\left(N=500, l_{p}=20 \sigma\right), i_{m c}$ and $i_{m d}$ are located within the physically knotted region about $60 \%$ and $85 \%$ of the times, respectively (Fig. 2b). We highlight that both these biases violate detailed balance because they systematically move TopoII to $i_{m c}$ and $i_{m d}$ without any intermediate steps. These models can thus be interpreted as extreme realisations of thermodynamic biases due to, for instance, conformation-driven docking [24] and transient enrichment in locally folded regions due to facilitated diffusion $[14,21]$. Indeed, while on ideal uniform substrates the binding rate is given by the Smoluchowski constant $k_{o n}=4 \pi D_{3} b-$ with $D_{3}$ the three dimensional diffusion coefficient and $b$ the target size - within a coiled substrate this binding rate depends on the typical distance between neighbouring segments $l \simeq \rho^{1 / 3}$. Thus, on timescales shorter than the chain relaxation time, a TopoII with non-specific attraction to DNA will undergo a significant oversampling of large density regions before exploring the rest of the substrate [14]. In fact, permanently looped or crumpled regions may act as sinks for weakly sticky proteins searching their target [19].

In Fig. 2c we compare the relaxation of the knotting probability for these different mechanisms: static, random jumps or jumps biased to regions of maximum curvature or maximum density (with same jumping rate). Intriguingly, the fastest relaxation is achieved when TopoII preferentially binds to regions of maximum density (see Fig. 2d for the decay time). Measuring $\kappa_{t}$ and $\operatorname{Max}|A(-1)|$ confirms that biases towards regions of maximum curvature enhance the simplification efficiency by reducing the number of topology-changing operations while rendering them more accurate, i.e. both $\kappa_{t}$ and $\operatorname{Max}|A(-1)|$ decrease. On the other hand, dynamic binding to regions of maximum density yields a qualitatively different relaxation pathway. As shown in Fig. 2c-d, we find that, although the number of topology-changing operations per unit time $\kappa_{t}$ is much larger than for any of the other models, this feature is only accompanied by a mild increase in $\operatorname{Max}|A(-1)|$ with respect to the others. In other words, binding to regions of high local density facilitates frequent strand-crossing operations but this is not accompanied by a significant enhancement of the knot complexity; on the contrary, it appears that most of these operations are still simplifying the topology.

To better understand our findings we design an idealised 2D random walk model that explores a set of disks representing different knot spaces. Each space contains the set of polymer conformations with fixed topology and multiplied by the $N$ possible locations of TopoII on the polymer. The disks have quickly decreasing radii $R$ for increasing topological complexity (see Fig. 3 and SM). We start the random walks at the centre of the $5_{1}$ space (green disk) and compare four processes: (i) pure diffusion and with jumps which can be (ii) random, (iii) within the annulus and (iv) within the vertical strips clos- 


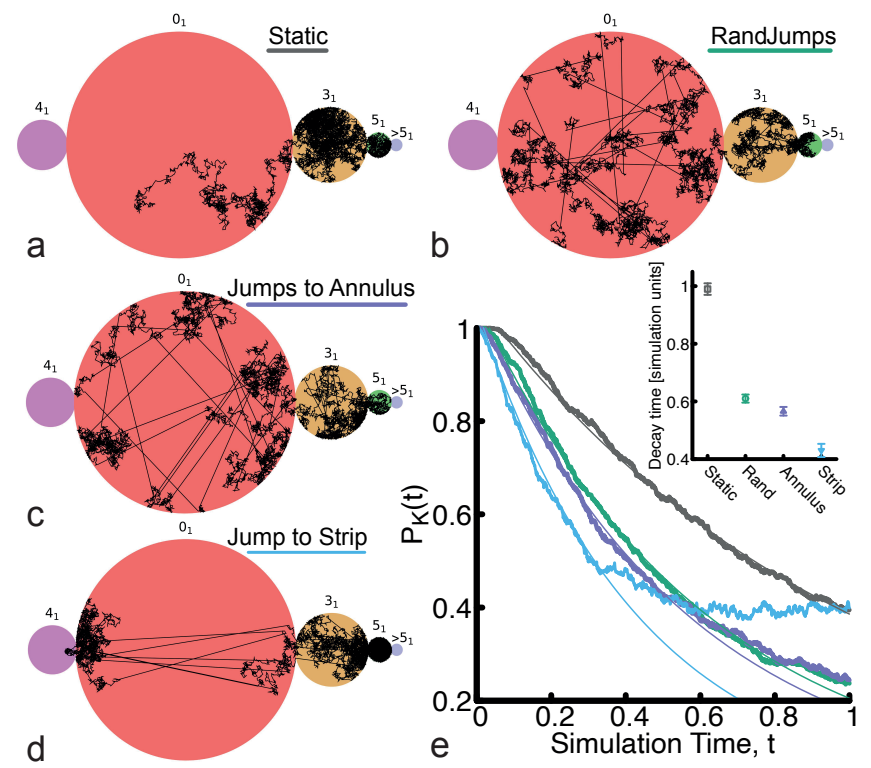

Figure 3. A simplified model of topological sampling. a-d Example trajectories of walks within simplified topological spaces: (a) purely diffusive and (b-d) with jumps either random or closer to the annulus or the vertical strip near the boundary of the disk. All simulations are initialised from within the $5_{1}$ space (green disk). e Shows $P_{K}(t)$ curves generated by this simplified model which well capture the faster relaxation for walks wit biased jumping. Solid lines show the fits an exponential function $e^{-t / \tau}$ at early times. The inset shows the decay time $\tau$.

est to the boundary of the disks. Each jump should be interpreted as an instantaneous change in the position of TopoII at fixed polymer conformation and therefore qualitatively associate (i) with static TopoII (ii) with random TopoII jumps and (iii-iv) with jumps of TopoII to regions of maximum curvature/density. This analogy is based on the assumption that the regions closest to neighbouring knot spaces are populated by conformations likely to precede a change in topology; among these, we argue there should be some in which TopoII is bound at regions of large local curvature/density. Thus, jumping to the annulus of the knot space or the vertical strip near the neighbouring space enhances the chances for the walker to make a topological transition. By comparing Fig. 3e with Fig. 2c-d, we find that these simple models can recapitulate our findings.

Finally, we investigate the sensitivity of dynamic models against substrate length $N$ and flexibility. In Fig. 4 we show that, unlike the static model of TopoII, all the dynamic models yield relaxation curves that are very weakly dependent of $N$ and persistence length. Additionally, from Fig. 4d it is apparent that biasing the binding dynamics towards region of maximum local density provides the most efficient way of performing topological relaxation, especially for very long substrates.

We explain this weak dependence on substrate length (reminiscent of some recent experiments in vivo finding plateauing knot complexity at large DNA lengths [2])
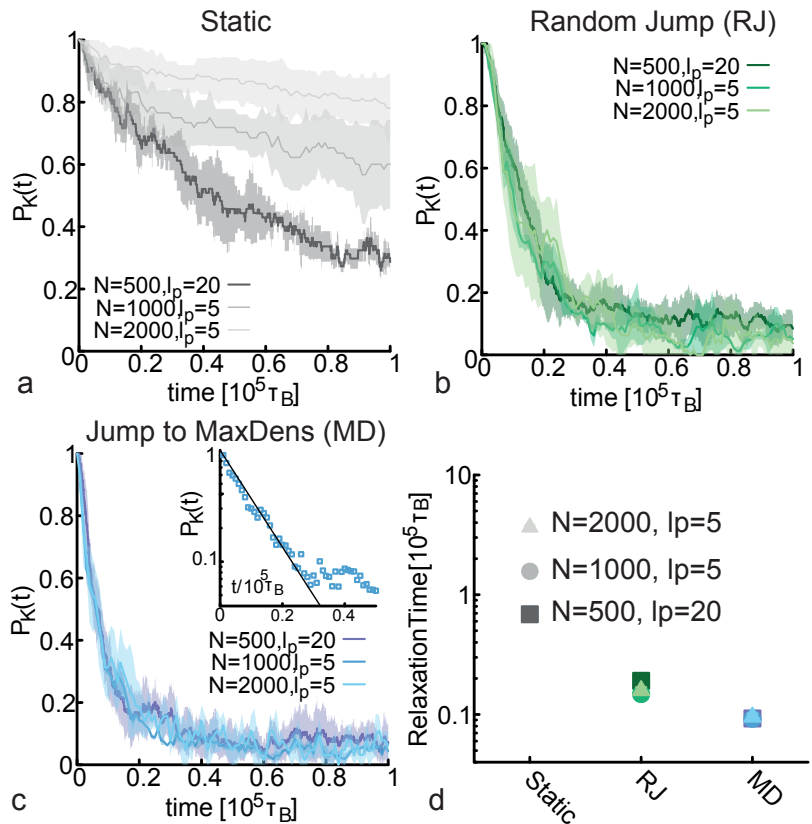

Figure 4. Dynamic Simplification Rate is independent on DNA Length. a Simplification curves for Static TopoII. b Simplification curves for dynamic random jump, rate $k_{j}=10^{-2} \tau_{B}^{-1}$. c Simplification curves for dynamic jump to max density, rate $k_{j}=10^{-2} \tau_{B}^{-1}$. Inset shows the exponential fit $e^{-t / \tau}$ of one of the curves at short times. $\mathbf{d}$ Topological relaxation timescale plotted for the different systems.

as follows. Physical knots tied on polymer chains display contour lengths $l_{k}$ that scale with the length of the chain $N$ as $l_{k} \sim N^{\theta}$ with $0<\theta<1$ in dilute, good solvent conditions and with $\theta \simeq 1$ under isotropic confinement $[15,16,25]$. For a static TopoII, the topological relaxation time is bound to be comparable to the (curvilinear) diffusion time of the knotted portion $\tau_{R} \sim\left(N-l_{k}\right)^{2} / D_{c} \sim\left(N-l_{k}\right)^{2} l_{k} / D_{0} \sim N^{2+\theta} / D_{0}$, in the limit $N \rightarrow \infty$ and where $D_{0}$ is the microscopic diffusion of a bead. On the other hand, a jumping TopoII only needs a timescale that scales as the (inverse) probability of landing on a segment that belongs to the knotted portion, i.e. $\tau_{T} \sim k_{j}^{-1}\left(N / l_{k}\right) \sim N^{1-\theta}$. Clearly, this is a much weaker scaling in polymer length $N$ with respect to the static case. Remarkably, the more delocalised the knot, the closer $\theta$ to unity (e.g. under strong isotropic confinement or in bad solvent $\theta \simeq 1[16,27])$ and the starker the difference in timescales, $\tau_{R} / \tau_{T} \sim N^{2 \theta+1}$.

Conclusions. Understanding how Topoisomerase regulate genome topology remains an open challenge in biophysics. Here we have studied previously underappreciated yet realistic aspects which may affect the action of TopoII on DNA: its dynamics and biased binding towards regions of large curvature or local density of the substrate. We discover that dynamic binding, which is realistic in high salt conditions, yields enhanced sampling of topological spaces which in turn drives faster topological simplification. Strikingly, introducing dynamic binding renders the topological relaxation virtually independent 
on the substrate length. To the best of our knowledge this aspect has never been tested experimentally and could be realised with time-resolved gel electrophoresis measurements of DNA topology in vitro. Introducing dynamic and biased binding may thus bring us closer to a full understanding of this fascinating problem.

DM is a Royal Society University Research Fellow and is supported by the ERC (Starting Grant, Topologically Active Polymers, Ref. 947918). LT acknowledges support from MIUR, Rita Levi Montalcini Grant, 2016. Source codes are available at https://git.ecdf.ed.ac.uk/ygutier2/topo2-lammpsimplementation.git.

* davide.michieletto@ed.ac.uk

† yair.fosado@ed.ac.uk

[1] V. V. Rybenkov, N. R. Cozzarelli, and A. V. Vologodskii, Proc. Natl. Acad. Sci. USA 90, 5307 (1993).

[2] A. Valdés, J. Segura, S. Dyson, B. Martínez-García, and J. Roca, Nucleic Acids Research 46, 650 (2018).

[3] D. Goundaroulis, E. Lieberman Aiden, and A. Stasiak, Biophysical Journal 118, 2268 (2019).

[4] J. T. Siebert, A. N. Kivel, L. P. Atkinson, T. J. Stevens, E. D. Laue, and P. Virnau, Polymers 9, 1 (2017).

[5] E. Orlandini, D. Marenduzzo, and D. Michieletto, Proceedings of the National Academy of Sciences 116, 8149 (2019).

[6] D. Racko, F. Benedetti, D. Goundaroulis, and A. Stasiak, Polymers 10, 1 (2018).

[7] A. Flammini, A. Maritan, and A. Stasiak, Biophys. J. 87, 2968 (2004).

[8] D. Michieletto, D. Marenduzzo, and E. Orlandini, Phys. Biol. 12, 036001 (2015).

[9] A. Vologodskii, New York, USA: Association for Computing Machinery, 266 (1998).
[10] A. V. Vologodskii, W. Zhang, V. V. Rybenkov, A. A. Podtelezhnikov, D. Subramanian, J. D. Griffith, and N. Cozzarelli, Proc. Natl. Acad. Sci. USA 98, 3045 (2001).

[11] J. Yan, M. O. Magnasco, and J. F. Marko, Nature 401, 932 (1999).

[12] Z. Liu, L. Zechiedrich, and H. S. Chan, Journal of molecular biology 400, 963 (2010).

[13] P. C. Bressloff and J. M. Newby, Reviews of Modern Physics 85, 135 (2013).

[14] M. A. Lomholt, B. D. Van Broek, S. M. J. Kalisch, G. J. Wuite, and R. Metzler, Proceedings of the National Academy of Sciences of the United States of America 106, 8204 (2009).

[15] B. Marcone, E. Orlandini, A. L. Stella, and F. Zonta, Journal of Physics A: Mathematical and General 38 (2005), 10.1088/0305-4470/38/1/L03.

[16] L. Tubiana, E. Orlandini, and C. Micheletti, Phys. Rev. Lett. 107, 1 (2011).

[17] S. Plimpton, J. Comp. Phys. 117, 1 (1995).

[18] L. Tubiana, E. Orlandini, and C. Micheletti, Prog. Theor. Phys. Suppl. 191, 192 (2011).

[19] C. A. Brackley, M. E. Cates, and D. Marenduzzo, Phys. Rev. Lett. 109, 168103 (2012).

[20] C. Loverdo, O. Bénichou, R. Voituriez, A. Biebricher, I. Bonnet, and P. Desbiolles, Phys. Rev. Lett. 102, 188101 (2009).

[21] L. Mirny, M. Slutsky, Z. Wunderlich, A. Tafvizi, J. Leith, and A. Kosmrlj, J. Phys. A 42, 434013 (2009).

[22] X. R. Bao, H. J. Lee, and S. R. Quake, Phys. Rev. Lett. 91, 265506 (2003).

[23] M. Doi and S. Edwards, The theory of polymer dynamics (Oxford University Press, 1988).

[24] A. H. Hardin, S. K. Sarkar, Y. Seol, G. F. Liou, N. Osheroff, and K. C. Neuman, Nucleic Acids Research 39, 5729 (2011).

[25] L. Tubiana, A. Rosa, F. Fragiacomo, and C. Micheletti, Macromolecules 46, 3669 (2013).

[26] A. Y. Grosberg and Y. Rabin, Physical Review Letters 99, 1 (2007).

[27] P. Virnau, Y. Kantor, and M. Kardar, Journal of the American Chemical Society 127, 15102 (2005). 